\begin{tabular}{|l|l|}
\hline & \\
\hline
\end{tabular}

DOSSIÊ

\title{
A complementaridade de pais e de profissionais na avaliação em intervenção precoce
}

\author{
The complementarity of parents and professionals in early intervention assessment \\ La complementariedad de padres y profesionales en la evaluación en la intervención temprana
}

\section{Ana Paula da Silva \\ Pereira $^{1}$ \\ orcid.org/0000-0003-4611-7602 \\ appereira@ie.uminho.pt}

\section{Andréa Perosa Saigh \\ Jurdi $^{2}$ \\ orcid.org/0000-0002-1111-5562 \\ andreajurdi@gmail.com}

Helena Isabel Silva Reis ${ }^{3}$

orcid.org/0000-0002-3589-8354 helenaisabelsilvareis@gmail.com

Recebido em: 14 ago. 2019. Aprovado em: 16 jan. 2020. Publicado em: xx xxx. 2020.

\section{(c) (i)}

Artigo está licenciado sob forma de uma licença Creative Commons Atribuição 4.0 Internacional.
Resumo: O estudo teve por objetivo analisar a convergência entre pais e profissionais relativamente à avaliação da área da interação social em crianças com Transtorno do Espectro Autista (TEA), entre três e seis anos, apoiadas pelo sistema de intervenção precoce em Portugal. A metodologia é de natureza quantitativa correlacional e a amostra foi constituida por 128 crianças com TEA. Os resultados concluem que os pais avaliam mais positivamente a dimensão da interação social comparativamente com os profissionais; a variável idade influenciou a avaliação de pais e de profissionais, verificando-se uma relação positiva entre as competências e o avanço da idade das crianças; e, as variáveis formação inicial, anos de experiência dos profissionais no apoio a crianças com TEA, nivel educacional e profissional dos pais não influenciaram os resultados ao nível da interação social. A investigação sugere a importância da intervenção precoce, priorizando as relações de colaboração entre pais e profissionais no processo de avaliação.

Palavras-chave: intervenção precoce, avaliação, pais, profissionais, transtorno do espectro do autismo, interação social.

Abstract: The aim of the study was to analyze the convergence between parents and professionals regarding the assessment of the social interaction dimension in children with Autism Spectrum Disorder (ASD), between the ages of 3 and 6 years, supported by the early intervention system in Portugal. The methodology applied was quantitative and the sample consisted of 128 children with ASD. The results show that: parents assess the social interaction dimension more positively compared to professionals; the variable ages influenced the assessment of parents and professionals, with a positive relationship between skills and the advancement of the children's age; the variables training, years of experience of professionals in supporting children with ASD, educational and occupation parents levels did not influence the results in social interaction dimension. Therefore, research suggests the importance of early intervention, and the collaborative relationships between parents and professionals in the assessment process.

Keywords: early intervention, assessment, parents, professionals, autism spectrum disorder, social interaction.

Resumen: La investigación tiene como objetivo analizar la convergencia entre padres y profesionales con respecto a la evaluación del área de interacción social en niños con TEA, entre los 3 y los 5 años de edad, apoyadas por el sistema de intervención precoz en Portugal. La metodologia es de naturaleza cuantitativa correlacional y la muestra ha sido constituida por 128 niños con TEA. Los resultados concluyen que los padres evalúan más positivamente la dimensión de la interacción social, comparados a los profesionales; la variable edad ha influenciado la evaluación de padres y de profesionales, comprobándose una relación positiva entre las competencias y el avance de la edad de los niños; las variables formación inicial, años de experiencia de los profesionales en el apoyo a niños con TEA, nivel educacional y profesional de los padres, no han influenciado los

\footnotetext{
Instituto de Educação da Universidade do Minho (IE-UM), Braga, Portugal.

Universidade Federal de São Paulo (UNIFESP), São Paulo, SP, Brasil.

Instituto Politécnico de Leiria (IPL), Leiria, Portugal.
} 
resultados al nivel de la interacción social. La investigación sugiere la importancia de la intervención precoz, priorizándose las relaciones de colaboración entre padres y profesionales en el proceso de evaluación.

Palabras clave: intervención precoz, evaluación, padres, profesionales, trastorno del espectro autista, interacción social.

\section{Introdução}

A avaliação da criança em Intervenção Precoce (IP) é um processo complexo que requer um conjunto de saberes de diferentes áreas disciplinares, bem como a participação ativa da familia em todo o processo (GrishamBrouw, Pretti-Frontczak, 2011; Wolraich, Gurwitch, \& Knight, 2005). A familia torna-se assim um elemento chave para a obtenção de informação fidedigna acerca do desenvolvimento da criança e da sua funcionalidade nos diferentes contextos (Hemmeter, Joseph, Smith, \& Sandall, 2001; Kuhaneck \& Watling, 2010).

A avaliação em IP é definida como um processo de colaboração entre profissionais e familia que deve: a) perspectivar um modelo integrado de desenvolvimento, que contemple uma visão holística e ecológica da criança e da sua família; b) basear-se em múltiplas fontes de informação e em múltiplas componentes, tendo em conta a complexidade do desenvolvimento, dos contextos e dos instrumentos; $\mathrm{c}$ ) basear-se no contexto das relações e das interações da criança e da familia; d) perspectivar uma relação de colaboração entre a família e os profissionais; e, e) constituir-se como o alicerce de todo o processo de intervenção e de apoio (Meisels \& Atkins-Burnett, 2000; Wolraich, Gurwitch, \& Knight, 2005)

Atualmente os profissionais e as familias têm promovido mudanças importantes na avaliação das crianças na IP, mudanças essas que resultam das evidências internacionais que sustentam as melhores práticas no processo de avaliação, nomeadamente o trabalho em equipe, a abordagem centrada na família e a valorização dos contextos de vida da criança e da família (Division of Early Childhood [DEC], 2014; Magalhães \& Pereira, 2013; Serrano \& Pereira, 2011).

O trabalho em equipe na avaliação possibilita diferentes perspectivas e diferentes conhecimentos (profissionais e familiares) que proporcionam uma visão mais autêntica do desenvolvimento, da aprendizagem e das necessidades da criança e das preocupações e prioridades da sua família. A avaliação deve ser, assim, convergente nas diferentes perspectivas, de famílias e profissionais, de modo a possibilitar uma melhor e mais adequada informação de base do desenvolvimento e dos estilos de aprendizagem da criança (Bagnato \& Levan, 2007: Reis, Pereira, \& Almeida, 2017).

A abordagem centrada na família, na avaliação preconiza a identificação das competências da criança e da família, respostas às suas preocupações e prioridades, e a individualização dos apoios e dos serviços, que devem ter em conta os valores, crenças e cultura da família. Essa abordagem considera toda a familia como unidade de intervenção, reconhecendo que o bem-estar de cada membro afeta o restante e baseia-se em estratégias de identificação e de resposta às preocupações da familia e não apenas às necessidades da criança (Dunst, Trivette, \& Hamby, 2007; Pereira \& Serrano, 2014).

Além disso, a abordagem centrada na familia considera que a familia deve ser um elemento ativo do processo de avaliação que pode escolher e decidir os diferentes papéis (observador, capacitador, e outros) que deseja adotar nesse processo. A familia, ao ser o elemento mais próximo da criança por excelência, é também o elemento mais capaz de obter melhores niveis de interação com a criança e alcançar o seu melhor nivel de funcionamento (Bagnato, 2007).

Os contextos naturais (lares, creches, jardins de infância, parques) são igualmente considerados fundamentais no processo de avaliação em IP. A avaliação deve, assim, contemplar os momentos de rotina da criança e sua familia, os materiais que fazem parte desses contextos e todas as pessoas que são familiares e significativas para a criança (Dunst, Bruder, \& Espe-Sherwindt, 2014; Bagnato, 2007; Mcwilliam, 2010).

São vários os autores que identificam a colaboração como uma das qualidades da 
avaliação, mencionando que os métodos e estilos de avaliação deverão promover o trabalho de equipe entre familias e profissionais, sendo os pais parceiros ativos na avaliação da criança (Bagnato, 2007; Bell, Corfield, David, \& Richardson, 2009).

A valorização da participação da família no processo de avaliação releva a necessidade de aprofundar formas e instrumentos de avaliação ajustados à diversidade das familias das crianças apoiadas na IP, instrumentos esses, que deverão contemplar a participação ativa e interativa dos profissionais e das familias de forma a tornar possivel o desenvolvimento de uma visão partilhada e de uma informação holística acerca da criança (Hall, Rutland, \& Grisham-Brown, 2011; M. Macy, Bagnato, R. Macy, \& Salaway, 2015; Serrano \& Pereira, 2011).

\section{Questão, objetivos e hipótese de investigação}

O estudo teve por objetivo analisar a convergência entre pais e profissionais relativamente à avaliação da área da interação social em criança com TEA, com idades compreendidas entre os três e os seis anos, residentes em Portugal continental e definiu como objetivos específicos:

(a) caracterizar do ponto de vista sociodemográfico e profissional os participantes no estudo (idade da criança e dos pais; gênero da criança; tipos de apoios prestados à criança; horas de apoio à criança; habilitações académicas, profissão, estado civil e idade dos pais; habilitações acadêmicas, formação inicial, e experiência dos profissionais em TEA);

(b) comparar a avaliação realizada pelos pais e pelos profissionais na área da interação social de crianças com TEA;

(c) e analisar comparativamente a percepção dos pais e dos profissionais acerca do desenvolvimento na área da interação social de crianças com TEA, considerando as seguintes variáveis: as habilitações acadêmicas e nivel socioeconômico dos pais, a formação inicial e a experiência na área das TEA dos pro- fissionais, o gênero e a idade da criança, bem como a condição de familiar e de profissional que apoia a criança.

As hipóteses do estudo foram definidas a partir da seguinte questão de investigação:

(a) em que medida existe diferenciação na percepção dos pais e profissionais acerca do desenvolvimento na área da interação social de crianças com TEA, com idades compreendidas entre os três e os seis anos, em Portugal continental?

No sentido de clarificarmos os objetivos e a questão de investigação definimos as seguintes hipóteses especificas:

H1. existem diferenças na avaliação dos pais e dos profissionais relativamente à área da interação social de crianças com TEA, com idades entre os três e os seis anos, residentes em Portugal continental;

H2. o nivel de escolaridade e o nivel profissional dos pais influenciam a avaliação da área da interação social de crianças com TEA, com idades entre os três e os seis anos, residentes em Portugal continental;

H3. a formação inicial dos profissionais tem influência na avaliação da área da interação social de crianças com TEA, com idades entre os três e os seis anos, residentes em Portugal continental;

H4. o gênero da criança influencia a avaliação dos pais e dos profissionais na área da interação social de crianças com TEA, com idades entre os três e os seis anos, residentes em Portugal continental;

H5. a idade da criança influencia a avaliação dos pais e dos profissionais na área da interação social de crianças com TEA, com idades entre os três e os seis anos, residentes em Portugal continental.

\section{Método}

A metodologia é de natureza quantitativa correlacional uma vez que procurou compreender os fenômenos através da formulação de hipótese sobre as relações entre as variáveis. Delineadas as hipóteses de investigação, foi possivel a 
identificação das variáveis, suas relações, bem como o papel que cada uma assumiu no estudo. A variável é um conceito operacional e classificatório, face a um objeto de conhecimento teoricamente importante, opera uma divisão em classes de equivalência mais ou menos relevantes (Almeida \& Freire, 2008).

Na elaboração do plano de investigação definiu-se que participariam no estudo, todos os pais de crianças diagnosticadas com TEA, com idades compreendidas entre os três e os seis anos, residentes em Portugal continental, bem como um dos profissionais que as atende. preferencialmente, aquele que fornece apoio há mais tempo a criança.

Para que a amostra do estudo tivesse o máximo de heterogeneidade contactou-se o maior número possivel de instituições e de serviços públicos e privados existentes em Portugal continental, em que se encontravam as crianças, objeto do estudo. Aproxima-se de uma amostra aleatória, uma vez que não houve qualquer intencionalidade na sua seleção, embora possa estar dependente da disponibilidade dos pais das crianças, bem como dos profissionais que as apoiam (Almeida \& Freire, 2008).

Foram entregues 441 questionários emparelhados, tendo sido devolvidos 274. Excluiram-se da amostra 146 crianças, por não ter sido exequivel o preenchimento do instrumento por ambas as partes, isto é, um dos seus pais e um dos profissionais que acompanhava a criança. A amostra do estudo foi constituida por 128 crianças diagnosticadas com TEA, com idades compreendidas entre os três e os seis anos, residentes em Portugal continental. Para cada criança, houve a participação de um dos seus pais, bem como de um dos profissionais que a apoiam. Deste modo, analisaram-se 256 questionários, relativos a 128 crianças, registandose um retorno de $29 \%$.

\section{Instrumento de coleta de dados}

Foi utilizada apenas a dimensão da avaliação da interação social da Escala de Avaliação do Perfil Desenvolvimental de Crianças com Perturbação do Espectro do Autismo (EACPEA), desenvolvida e validada em território português por Reis, Pereira e Almeida (2017). A dimensão da interação social da EACPEA é constituida por 23 itens.

O instrumento utiliza uma escala do tipo Likert, com 5 itens de resposta para cada pergunta, respectivamente: nunca ou quase nunca; poucas vezes; às vezes; bastante vezes; sempre ou quase sempre; e opção adicional sem informação (quando não é possivel observar o comportamento ou quando não se aplica à criança).

Em relação aos dados sóciodemográficos foi utilizado um questionário que fez referência à informação socioprofissional dos sujeitos participantes.

\section{Análise dos dados}

No tratamento dos dados recolhidos utilizouse a análise estatística, recorrendo-se à utilização de modelos de estatística descritiva e inferencial. Procedemos à codificação das variáveis dependentes e independentes do nosso estudo, e em seguida, introduzimos e analisamos através software aplicativo de caráter cientíico, Statistical Package for the Social Sciences (SPSS), na versão 22, para Windows.

\section{Apresentação dos resultados descritivos da amostra}

A distribuição dos dados da amostra, relativamente à zona de residência da criança, foi ponderada em cinco regiões de Portugal continental. Verificou-se que, do total das crianças $59 \%(n=76)$ residem na Zona Norte, $25 \%(n=32)$ na Zona Centro, 13\% ( $n=17$ ) em Lisboa e Vale do Tejo, 2\% (=2) no Alentejo e 1\% ( $n=1)$ no Algarve.

Em relação ao gênero permite-nos verificar que do grupo total das crianças participantes $(\mathrm{N}=128)$, $86 \%(n=110)$ são do gênero masculino e $14 \%$ ( $n=18)$ são do gênero feminino. No que concerne à faixa etária das crianças da amostra, constatou-se que a média de idades das crianças é de 56 meses.

Quanto à idade dos pais das crianças, verificouse que a média é de 38 anos, sendo o mínimo de 27 e o máximo de 53 anos de idade. Referente à idade das mães, a média das idades é inferior à dos pais, sendo de 36 anos. 
Em relação às habilitações acadêmicas dos pais das crianças, constatou-se que as mães e os pais apresentam em média, entre $05^{\circ}$ ano e o 9. ${ }^{\circ}$ ano de escolaridade.

A amostra total dos profissionais respondentes foi constituida por 128 elementos, cuja particularidade reside no facto de apoiarem a criança incluida no estudo. Constatou-se que 90\% ( $n=115)$ desses profissionais são do sexo feminino, sendo os restantes $10 \%$ ( $n=13$ ), do sexo masculino.

No que concerne à formação inicial dos profissionais respondentes, procedeu-se ao agrupamento em quatro áreas técnicas, nomeadamente: terapia ocupacional; terapia da fala; psicologia; e educação de infância.

Pôde se constatar que do total da amostra ( $\mathrm{N}=128)$, $44.5 \%$ dos profissionais ( $n=57$ ) possuem formação inicial na área da educação de infância, 19.5\% em psicologia ( $n=25), 19,5 \%$ em terapia ocupacional (25) e $16,4 \%$ na área da terapia da fala (21).

No que se refere ao tempo de experiência dos profissionais respondentes, quanto à intervenção com as crianças diagnosticadas com TEA, concluiu-se que, a amplitude da variável é significativa, podendo variar em o mínimo de 1 ano e um máximo de 20 anos, apresentando uma média de 6,5 anos de experiência.

\section{Análise e discussão dos resultados inferenciais}

Os resultados serão apresentados levando em consideração as questões da pesquisa e as respectivas hipótese de investigação.

Hipótese 1. Existem diferenças na avaliação dos pais e dos profissionais relativamente à área da interação social de crianças com TEA, com idades compreendidas entre os três e os seis anos de idade, residentes em Portugal continental. Verificouse que existem diferenças estatisticamente significativas na avaliação realizada pelos pais e pelos profissionais relativamente ao desenvolvimento de competências na área da interação social da criança. As respostas dos pais apresentam uma mediana de 2,95 e os profissionais 2,59. Desse modo, conclui-se que, a posição de pai (ou mãe) e de profissional que apoia a criança revela influência na avaliação do desempenho da criança com TEA, na área da interação social, sendo os pais a pontuar as crianças mais favoravelmente, em comparação com os profissionais

Esta situação vem ao encontro do que é evidenciado pela literatura, pela anunciada tendência crescente da participação das familias no processo de avaliação das crianças em IP. Converge desse modo, com o trabalho de Dunst (2002), na medida em que o autor considera importante o envolvimento e a participação dos pais nesse processo, evitando a dependência destes para com os profissionais. Há a ideia de que os pais não estão muito habituados a serem considerados parte incluida da equipe que trabalha com crianças, em IP (Pimentel, 2004). Porém, Reis, Pereira e Almeida (2017) mencionam o fato de alguns profissionais criarem oportunidades para que as famílias possam avaliar as crianças utilizando para tal, escalas de desenvolvimento ou escalas de verificação acessiveis e culturalmente ajustadas

Essa participação ativa dos pais confirma de igual modo as recomendações inerentes aos modelos de avaliação atuais, na medida em que mencionam que o processo de avaliação deverá ser realizado pelos indivíduos que sejam familiares às crianças, entre os quais, se destacam, os pais, os educadores e outros profissionais, possibilitando chegar ao melhor nivel de funcionamento da criança (Bagnato, 2007). A importância da avaliação se centrar na funcionalidade dos comportamentos potencializa a sua aquisição e generalização (Serrano \& Pereira, 2011; Mcwilliam, 2003).

Quando se aborda o tema da avaliação das crianças com TEA, a literatura apresenta um conjunto de instrumentos formais como possibilidade de recurso, no entanto, a avaliação concretizada pelos pais e pelos profissionais no âmbito do presente estudo, responde ao alerta lançado por Lima (2012), Hortal et al. (2011), pois consideram que os instrumentos formais apenas possibilitam aferir uma visão mais geral acerca do desenvolvimento da criança. Nesse sentido, 
é importante a articulação com a familia para desenvolvimento de um trabalho conjunto, na medida em que a avaliação realizada assume um papel orientador no desenvolvimento do plano de intervenção (Mcwilliam, 2003; Reis, Pereira \& Almeida, 2017; Serrano \& Pereira, 2011).

Retomando ao tema da participação ativa dos pais no processo de avaliação dos seus filhos, importa também considerar que é um momento relevante no conhecimento das aquisições da criança, de modo que possam estar mais capazes de proporcionar oportunidades de desenvolvimento. A participação ativa possiblita às famílias um sentimento de autoconfiança e no reforço das suas competências, configurando-se elementos privilegiados na promoção de novas oportunidades de desenvolvimento às suas crianças, tal como defendido por Lopes et al. (2009).

Para finalizar a evidência da participação dos pais no processo de avaliação das crianças, Siegel (2008) defende que, à medida que os pais adquirem novos conhecimentos acerca do comportamento e das competências dos seus filhos tornam-se mais capazes e envolvidos no seu processo de desenvolvimento. A análise complementar, possibilitou verificar que os pais avaliaram mais favoravelmente as crianças, quando comparada com a avaliação dos profissionais. A situação poderá ir ao encontro dos diversos contributos teóricos que atribuem distinta importância aos contextos, em as aprendizagens das crianças são cimentadas nas suas rotinas diárias, sendo os pais os privilegiados mediadores desses processos (Dunst et al., 2012). É esperado que a família confira à criança um meio acolhedor, protetor e que seja capaz de responder às necessidades socioemocionais nesses primeiros anos de vida, e nesse sentido, tenham também oportunidades privilegiadas para observarem e discernirem acerca do comportamento da criança (Rodrigues, 1997).

Aliás, e como já anteriormente evidenciamos, é importante que a observação aconteça no seu cotidiano (Bagnato, 2007), e, pelo recurso à avaliação funcional, permite um real cenário do comportamento da criança (Serrano \& Pereira, 2011). Neste sentido, os profissionais encontram- se em desvantagem, pois, à partida, observam a criança em contextos específicos de apoio e, em média, durante 3 horas por semana (Swanson, Raab, \& Dunst, 2011).

Finalizamos a discussão da hipótese 1 referindo que, a observação de comportamentos está inerente um caráter particular e individual (Schmidt, 2012). O autor defende que o processo de avaliação poderá ser influenciado pela apreensão e pela interpretação dos comportamentos de quem está a observar a criança. Neste sentido, entende-se, também, que a observação dos comportamentos das crianças não seja convergente entre todos, pais e profissionais.

Hipótese 2. O nivel educacional e o nivel profissional dos pais influência a avaliação da área da interação social de crianças com TEA, com idades compreendidas entre os três e os seis anos, residentes em Portugal continental. Verificou-se que não existem diferenças estatisticamente significativas na avaliação realizada pelos pais. Isso quer dizer que, o nivel educacional dos progenitores ( $p=.378$ ), bem como o seu nivel profissional ( $p=.994)$ não se revelam fatores influentes no processo de avaliação do desenvolvimento da criança, na área específica em estudo, a interação social.

Em análise complementar, observamos as medianas dos três niveis educacionais, designadamente: até ao $9 .^{\circ}$ ano, mediana de 2,40; $10 .^{\circ}$ ano ao $12 .^{\circ}$ ano, mediana de 2,50; e formação superior, com mediana de 2,54. Assim, verificamos uma ligeira tendência positiva, quer isto dizer que quanto mais elevadas forem as habilitações acadêmicas, mais favoráveis são as pontuações conferidas às crianças, embora essa evidência não confira significância estatística.

Olhando os dados obtidos, não se observaram diferenças estatisticamente significativas na avaliação da área de interação social das crianças com TEA, considerando as influências das variáveis nivel educacional e nivel profissional. Desse modo, os resultados não confirmam as conclusões da investigação de King e Bearman (2011), na medida em que esses conferem que a profissão e as habilitações acadêmicas das famílias das 
crianças com TEA são fatores influenciadores das oportunidades proporcionadas na vida das crianças. O estudo concluiu que as crianças com TEA que pertencem a familias com estatuto socioeconômico mais elevado apresentam mais possibilidades de acesso, não só à saúde, como à educação. Nesse sentido, esperavam-se diferenças entre as familias, isto é, que as crianças com TEA provenientes de familias com nivel profissional e escolar mais elevado apresentassem pontuações mais positivas, na medida em que poderiam se beneficiar de maiores oportunidades de aprendizagem, de desenvolvimento, assim como no acesso a apoios especializados.

Hipótese 3. A formação inicial dos profissionais influência a avaliação do desenvolvimento na área da interação social de crianças com TEA, com idades compreendidas entre os três e os seis anos, residentes em Portugal continental. Verificouse que a formação inicial dos profissionais que apoiam a criança não influencia nos resultados da avaliação do desenvolvimento da interação social das crianças com TEA.

O fato de não se terem identificado diferenças estatisticamente significativas entre os diversos profissionais poderá evidenciar a mais-valia do trabalho em equipe realizado nas equipes de IP em Portugal.

Segundo a perspetiva de Cartmill, Soklaridis, e Cassidy (2011), a abordagem em equipe proporciona a aprendizagem mútua entre os profissionais e uma troca flexivel de papéis entre os mesmos. Sendo que, essa abordagem, para além de promover e capacitar a criança e a sua familia, potencializa o estabelecimento de laços de confiança entre os diferentes elementos da equipe (Butt \& Caplan, 2010).

Hipótese 4. O gênero da criança influencia a avaliação, de pais e profissionais na área da interação social de crianças com TEA, com idades compreendidas entre os três e os seis anos, residentes em Portugal continental. Verificouse que existem diferenças estatisticamente significativas $(p \leq .05)$ na avaliação da área de interação social das crianças com TEA, quando realizada pelos progenitores e, considerando o gênero da criança $(p=.03)$. Desse modo, concluimos que os pais pontuam distintamente as crianças, meninos e meninas. Essa situação não é verificada na avaliação concretizada pelos profissionais, realizando-a sem que haja essa distinção, estatisticamente relevante, na observação do desempenho da criança na área da interação social $(p=.74)$.

Em análise complementar, observou-se as medianas das respostas dos pais, concluindo que os meninos têm uma mediana de 2,40 e as meninas 3,40. Isso quer dizer que as meninas obtiveram pontuações mais positivas, em que os pais consideram que as competências na área da interação social acontecem em uma frequência entre o 3 (às vezes) e o 4 (bastantes vezes). Relativamente aos rapazes, os pais conferiram frequências entre 2 (poucas vezes) e 03 (às vezes).

A literatura vem evidenciando diferenças nas características apresentadas por meninos e meninas, havendo variação dessas caracteristicas de criança para criança (Filipe, 2012; Lima, 2012; Paasche, Gorril, \& Strom, 2010).

Um estudo desenvolvido por Oliveira, Frizzo e Marin (2000) concluiu que as mães de crianças em idades pré-escolares são propensas a desenvolver atitudes diferenciadas para com os meninos e para com as meninas, explicando que, o gênero da criança é um fator mais ou menos explicativo das atitudes maternas de irritabilidade, rejeição e intrusão. Braz e Salomão (2002) realizaram um estudo que teve por base a perspectiva da interação social, e concluiram que as mães revelam diferenças nos seus comportamentos comunicativos para com meninos e meninas. As mães dos meninos apresentaram mais "diretivos de atenção" e as mães das meninas mais "diretivos de instrução". No que se refere às interações entre criança e mães, nos momentos da escolha do brinquedo, o gênero da criança determinava o tipo de brinquedo a usar.

O estudo desenvolvido por Pacheco, Silveira, e Schneider (2008) aponta a análise dos estilos parentais, assumindo portanto implicações desse fator no desenvolvimento das crianças. Esses autores apoiaram-se na opinião de diversos 
investigadores, concluindo que há pais que podem apresentar uma postura mais exigente quanto aos comportamentos dos filhos, ao invés de outros que optam por posições de menos exigência. Os autores citam os contributos de Repopold, Pacheco, e Hurtz (2005) para evidenciar que os estilos parentais estão assentes nas crenças, nos valores e nos aspetos relacionados com os papéis familiares. Essa ideia remete-nos à possibilidade de estarmos perante pais com estilos parentais próprios, cuja evidência se remete aos resultados estatisticamente significativos ao avaliarem o desempenho da criança na área da interação social.

Hipótese 5. A idade da criança influencia a avaliação, de pais e profissionais na área da interação social de crianças com TEA, com idades compreendidas entre os três e os seis anos, residentes em Portugal continental. Verificouse que existem diferenças estatisticamente significativas ( $p<.05)$ na avaliação concretizada pelos pais e pelos profissionais na área da interação social das crianças. Concluimos que os pais avaliam distintamente as crianças em conformidade com a sua idade $(p=.021)$. Essa realidade foi também verificada nas avaliações dos profissionais, apresentando diferenças com maior grau de significância estatística ( $p=.006)$.

\section{Considerações finais}

O estudo teve como finalidade analisar a convergência entre pais e profissionais relativamente à avaliação da área da interação social em crianças com TEA, com idades compreendidas entre os três e os seis anos.

A investigação na área dos TEA sugere que sejam dirigidas intervenções o mais precocemente possivel, como forma de potencializar os niveis de funcionalidade da criança. Aponta-se, por isso, para que a intervenção inicie após o diagnóstico, com recurso a avaliações interdisciplinares, programas individualizados e direcionados a todos os contextos de vida da criança (Paynter et al., 2012). Inerente ao apoio da IP está também o apelo à criação de relações de colaboração entre os profissionais e os pais, do mesmo modo que incitam à participação ativa dos pais em todo o processo de intervenção (Bagnato, 2007; Dunst, 1997; Dunst et al., 2012; Pimentel, 2004; Serrano \& Pereira, 2011). Nesse sentido, consideramos que este trabalho vem desde logo dar evidência à opinião de Dunst (2012), na medida em que esse se refere a uma tendência crescente dos profissionais para implementarem abordagens colaborativas e participativas conjuntamente com os pais.

A nossa investigação verificou que a condição de familiar (pai ou mãe) e de profissional que apoia a criança com TEA revela influência na avaliação do desempenho da criança na área da interação social. Concluímos, também, que os familiares avaliam mais favoravelmente o desempenho das crianças quando comparado com as pontuações dos profissionais.

O estudo concluiu, ainda, que o nível profissional e o nivel educacional dos pais não se evidenciaram determinantes na avaliação da dimensão da interação social das crianças com TEA. Seria esperado que a profissão e as habilitações acadêmicas dos pais interferissem nas oportunidades das crianças e, consequentemente, no desenvolvimento das mesmas, no entanto tal fato não foi verificado pelo estudo (King \& Bearman, 2011). A investigação verificou que os pais avaliam distintamente as crianças, meninos e meninas. Percebe-se que as crianças do gênero feminino obtiveram pontuações mais positivas do que as do gênero masculino. A literatura evidencia diferenças quanto à manifestação das características das crianças com TEA, pela frequência, severidade, condicionada também pelo nivel de desenvolvimento de cada criança e sua idade cronológica (American Psychological Association [APA], 2013), facto confirmado no nosso estudo. São também aportados ao gênero um conjunto de características e, por isso, aguardavam-se diferenças nos comportamentos dos meninos e das meninas.

Constatou-se, também, que os pais e os profissionais avaliam as crianças de forma distinta em conformidade com a idade dessas. Foi possivel constatar que as crianças mais novas obtiveram pontuações menos positivas por comparação com as conferidas às crianças mais velhas. Neste sentido, detectou-se uma relação positiva entre 
os comportamentos apresentados e o avanço da idade da criança. Considera-se que à medida que a criança vai sendo envolvida em interações sociais no seu dia a dia, com os outros e com o meio, aportam a si mesmas maiores benefícios, a curto e a longo prazo, e neste sentido se regista uma evolução positiva do desenvolvimento à medida que cresce, evidente pelas melhores competências demonstradas (Lyra \& Moura, 2000; Rapin \& Tuchaman, 2006).

Concluímos que o desenvolvimento das crianças com TEA tem sido apoiado não só pelos motivos apresentados no ponto anterior, mas também pela crescente consciencialização do constructo, não só pelos pais, mas também pelos profissionais, bem como pelos serviços especializados que são criados e desenvolvidos, como é exemplo o Sistema Nacional de Intervenção Precoce em Portugal (Decreto-Lei n. ${ }^{\circ}$ 281/2009), que perspetiva a universalidade no acesso, o direito à participação e à inclusão das crianças com necessidades especiais e suas famílias.

Apontamos à concretização do estudo algumas limitações. O estudo baseou-se na recolha de informação com uma escala em processo de construção e de adaptação, não podendo ser assegurado no final do estudo, que todos os itens analisados sejam importantes para a análise da dimensão da interação social nas crianças com TEA com idades entre os três e os seis anos.

Outro fator considerado limitativo prende-se com o tamanho da amostra. Considera-se que o fato de um dos critérios de participação no estudo considerar a necessidade de emparelhamento dos participantes, pai ou mãe e profissional que os apoia, constituiu se como um fator limitativo levando à exclusão de aproximadamente 50\% dos questionários recebidos.

\section{Agradecimentos}

Este trabalho é financiado pelo ClEd Centro de Investigação em Educação, projeto UID/CED/01661/2019, Instituto de Educação, Universidade do Minho, através de fundos nacionais da FCT/MCTES-PT.

\section{Referências}

Ameida, L., \& Freire, T. (2008). Metodologia da Investigação em Psicologia da Educação. Braga: Psiquilibrios Edições.

Bagnato, S. (2007) Authentic assessment for early childhood intervention: Best practices. New York: The Guilford Press.

Bagnato, S., \& Levan, R. (2007). Can professionals "Test without Tests" for authentic assessment?. In S. Bagnato (Ed.), Authentic Assessment for Early Childhood Intervention (pp. 98-116). New York: The Guildford Press.

Braz, F., \& Salomão, N. (2002). A fala dirigida a meninos e meninas: Um estudo sobre o input materno e suas variações. Psicologia: Reflexão e Critica, 15(2), 333-344 https://doi.org/10.1590/S0102-79722002000200011.

Butt, L., \& Caplan, B. (2010). The Rehabilitation Team In R. Frank, M. Rosenthal \& B. Caplan (Eds.), Handbook of Rehabilitation Psychology (pp. 451-457). Washington: American Psychological Association. https://doi.org/10.1037/15972-032.

Cartmill, C., Soklaridis, S., \& Cassidy, D. (2011). Transdisciplinary teamwork: The experience of clinicians at a functional restoration program. Journal of Occupational Rehabilitation, 21(1), 1-8. https://doi. org/10.1007/s10926-010-9247-3.

Division for Early Childhood. (2014). DEC recommended practices in early intervention/early childhood special education 2014. http://Ww/w.dec-sped.org/ recommendedpractices.

Dunst, C. (2000). Revisiting "rethinking early intervention. Topics in Early Childhood Special Education. 20(2), 95-104. https://doi.org/10.1177/027112140002000205.

Dunst, C. J. (2007). Early intervention for infants and toddlers with developmental disabilities. In Odom, S. L., Homer, R. H., Snell, M., \& Blacher J. (Eds.), Handbook of developmental disabilities (pp. 161-180). New York: Guilford Press.

Dunst, C., \& Bruder, M.B. (2002). Valued outcomes of service coordination, early intervention and natural environments. Exceptional Children, 68(3), 361-375. https://doi.org/10.1177/001440290206800305.

Dunst, C., Raab, M., Trivette, C., \& Swanson, J. (2012). Oportunidades de aprendizagem para a criança no quotidiano da comunidade. In R. Macwilliam (Ed.), Trabalhar com as familias de crianças com necessidades especiais (pp. 73-96). Porto: Porto Editora.

Dunst, C., Trivette, C., \& Deal, A. (1988). Enabling and Empowering Families: principles and guidelines for practice. Cambridge, MA: Brookline Books.

Dunst, C., Bruder, M.B., \& Espe-Sherwindt, M. (2014). Family Capacity-Building in Early Childhood Intervention: Do Context and Setting Matter?. School Community Journal. 24(1), 37-48.

Filipe, C. (2012). Autismo - conceitos, mitos e preconceitos. Lisboa: Verbo

Grisham-Brown, J., \& Pretti-Frontckzak (2011). Assessing Young Children in Inclusive Settings: the blended practices approach. Baltimore: Paul Brooks. 
Hortal, C., Bravo, A., Mitja, S., \& Soler, J. (2011). Alumnado com transtorno del espectro autista. Barcelona: Editorial GRAÓ.

King, M., \& Bearmen, P. (2011). Socioeconomic status and the increased prevalence of autism in California. Americam Sociological Review, 76(2), 320-346. https://doi.org/10.1177/0003122411399389.

Lima, C., \& Levy, P. (2012). A causa do autismo: Investigação etiopatogénica. In C.B. Lima (Ed.), Perturbações do Espectro do Autismo- manual prático de intervenção (pp. 13-22). Lisboa: LIDEL.

Lopes, R.C., Vivian, A., Oliveira, D., Silva, C., \& Piccinni, C.A. (2009). Quando eles crescem, eles voam: Percepções e sentimentos maternos frente ao desenvolvimento infantil aos 18-20 meses. Psicologia em Estudo, 14(2), 221-232. https://doi.org/10.1590/S141373722009000200002 .

Lyra, M.C., \& Moura, M.L.(2000). Desenvolvimento na interação social e no contexto histórico-cultural: adequação entre perspectiva teórica e metodologia Psicologia: Reflexão e Crítica, 13(2), 62-71. https://doi. org/10.1590/S0102-79722000000200002.

Macy, M., Bagnato, S., Macy, R., \& Salaway, J. (2015) Conventional tests and testing for early intervention eligibility. Is there an evidence base? Infants \& Young Children, 28(2), 182-204. https://doi.org/10.1097/ IYC.0000000000000032.

Magalhães, S., Pereira, A.P. (2013). A Avaliação do Desenvolvimento em Intervenção Precoce: perceções das familias portuguesas. Revista Galego-Portuguesa de Psicoloxia e Educacion, 21(1), 149-164.

Mcwilliam, R. (2010). Routines-based Early Intervention. Baltimore, MD: Paul H. Brooks Publishing Co.

Mcwilliam, P. (2003). Repensar a avaliação da criança. In P. Mcwilliam, P. Winton, \& E. Crais (Eds.), Estratégias Práticas para a Intervenção Precoce Centrada na Familia (pp. 65-79). Porto: Porto Editora.

Oliveira, E., Frizzo, G., \& Marin, A. (2000). Atitudes maternas diferenciais para meninos e meninas de quatro e cinco anos. Psicologia: Reflexão e Critica. 13(3), 363-371. https://doi.org/10.1590/S010279722000000300005 .

Paasche, L., Gorrill, L., \& Storm, B. (2010). Crianças com Necessidades Especiais em Contextos de Educação de Infância. Porto: Porto Editora.

Pacheco, J., \& Silveira, L. (2008). Estilos e práticas educativas parentais: análise da relação desses construtos sob a perspectiva dos adolescentes Psicologia, 39(1), 66-73.

Paynter, J., Scott, J., Beamish, W., Duhig, M., \& Heussler, $H$. (2012). A Pilot study of the effects of an australian centre-based early intervention program for children with autism. Open Pediatric Medicine Journal, 6, 7-14. https://doi.org/10.2174/1874309901206010007.

Pereira, A.P., \& Serrano, A.M. (2014). Early Intervention in Portugal: Study of professionals perceptions. Journal of Family Social Work, 17(3), 263-282. https:// doi.org/10.1080/10522158.2013.865426.
Pimentel, J. (2004). Avaliação de programas de intervenção precoce. Análise Psicológica, 1(XXII), 43-54

Reis, H., Pereira, A.P., \& Almeida, L. (2013). Construção e validação de um instrumento de avaliação do perfil desenvolvimental de crianças com perturbação do espectro do autismo. Revista Brasileira de Educação Especial, 19(2),183-194. https://doi.org/10.1590/ S1413-65382013000200004.

Reis, H., Pereira, A.P., \& Almeida, L. (2018). Intervention Effects on Communication Skills and Sensory Regulation on Children with ASD. Journal of Occupational Therapy, Schools, \& Early Intervention,11(3), 346359. https://doi.org/10.1080/19411243.2018.1455552.

Scmidt, C. (2012). O autismo: Uma análise do filme. Revista Brasileira Educação Especial, 18(2), 179-194. https://doi.org/10.1590/S1413-65382012000200002.

Serrano, A. M., \& Pereira, A. P. (2011). Parâmetros Recomendados para a Qualidade da Avaliação em Intervenção Precoce. Revista Educação Especial, 24(40), 63-180.

Siegel, B. (2008). O Mundo da Criança com Autismo: Compreender e tratar perturbações do espectro do autismo. Porto: Porto Editora.

Swanson, J., Raab, \& M., Dunst, C. (2011). Strengthening Family Capacity to Provide Young Children Everyday Natural Learning Opportunities. Journal of Early Childhood Research, 9(1), 66-80. https://doi. org/10.1177/1476718X10368588.

\section{Ana Paula da Silva Pereira}

Doutora em Educação Especial pela Universidade do Minho (UM, Braga, Portugal), investigadora do Centro de Investigação em Educação (ClEd), Professora do Departamento de Psicologia da Educação e Educação Especial do Instituto de Educação da Universidade do Minho, Braga, Portugal.

\section{Andréa Perosa Saigh Jurdi}

Pós Doutorada em Estudos da Criança pelo Instituto de Educação da Universidade do Minho (UM, Braga, Portugal), Professora Associada do Curso de Terapia Ocupacional e do Departamento Saúde, Educação e Sociedade da Universidade Federal de São Paulo, Brasil.

Helena Isabel Silva Reis

Doutorada em Estudos da Criança pelo Instituto de Educação da Universidade do Minho (UM, Braga, Portugal), Professora na Escola Superior de Saúde de Leiria (ESSLei) no Politécnico de Leiria, Leiria, Portugal.

\section{Endereço para correspondência}

Andréa Perosa Saigh Jurdi

Universidade Federal de São Paulo, SP

Rua Loefgren, 1984 - Vila Clementino, São Paulo - SP, 04040-003 